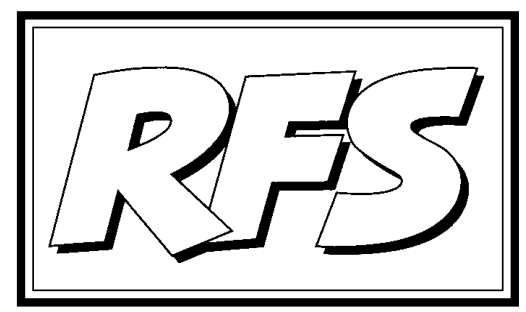

Revista de Fomento Social, 58 (2003), 525-533

\title{
Comentarios sobre el Plan Económico Andalucía Siglo XXI
}

\section{-Adolfo RODERO FRANGANILLO² y Ma del Carmen LÓPEZ MARTíN²-}

(Palabras clave: Planificación, Andalucía, comparación de planes. Key Words: Planning, Andalusia, Comparison of Plans)

Recientemente ${ }^{3}$ se ha publicado el Plan Económico Andalucía Siglo XXI 2002-20054 . Es el quinto plan económico aprobado por el Parlamento de

1 Estos comentarios se refieren esencialmente al proceso planificador andaluz, si bien se incluyen deforma puntual alusiones a la publicación que recoge el Plan Económico Andalucía Siglo XXI. No se ha pretendido, por tanto, realizar una mera recensión de un libro editado.

2 Facultad de CC. EE. y Empresariales - ETEA, adscrita a la Universidad de Córdoba.

3 El Plan fue aprobado los días 13 y 14 de noviembre de 2002; en la edición, el depósito legal es de 2003.

4 Este extenso libro (542 páginas de tamaño folio) comprende los trece capítulos siguientes: 1ㅇ) Fundamentos de la planificación en Andalucía; 2ㅇ) La economía andaluza en su entorno; 3우 Factores para una competitividad sostenible; 4ㅇ) Perspectiva sectorial de la economía andaluza; 5o) Estrategia de desarrollo; 60) Políticas de tejido productivo; 70) Políticas de empleo y capital 
Andalucía desde que se creó la comunidad autónoma (CA) andaluza 5 . Podemos afirmar, por tanto, que la planificación tiene ya una larga tradición en nuestra CA. ${ }^{6}$

\section{Objetivos de los planes andaluces}

Para analizar los objetivos del último plan, puede ser interesante recordar cómo ha evolucionado la fijación de objetivos en los cinco planes (ver cuadro página siguiente).

Lo primero que sorprende en dicha relación es la falta de precisión. Si bien los objetivos finales aparecen claramente en los planes, los intermedios figuran de forma mucho menos precisa; unas veces se denominan efectivamente objetivos intermedios, otras veces objetivos operativos y otras se incluyen como estrategias de desarrollo. En parte estas diferencias responden a contenidos distintos; sin embargo, los incluimos en una sola columna.

Los objetivos finales se mantienen a lo largo de los años, centrándose sobre todo en la creación de empleo y de forma menos evidente en la redistribución de la renta o de la riqueza. Este enfoque es coherente con los principales problemas de la economía andaluza. También hay que valorar positivamente que se mantengan los objetivos para que no se produzca una ruptura desaconsejable de la política planificadora.

Algo más variable y falta de coherencia es la determinación de lo que hemos llamado objetivos intermedios. Si de un plan a otro se producen cambios profundos de objetivos difícilmente se pueden conseguir resultados duraderos. La planificación es en gran parte una política a medio o largo plazo que exige la permanencia de las decisiones de política, para que éstas puedan

humano; 8) Políticas de factores básicos de competitividad; 9ㅇ) Políticas de cohesión social; 10을 Políticas de ordenación y equilibrio territorial; 11으) Políticas de sostenibilidad ambiental; 12ㅇ) Escenario financiero y programación económica; 13o) Sistema de seguimiento, evaluación y control del Plan económico Andalucía siglo XXI.

5 En la bibliografía se relacionan los cinco planes de desarrollo de la comunidad andaluza.

6 El artículo 18.1 del Estatuto de autonomía para Andalucía indica que "corresponde a la Comunidad autónoma andaluza, de acuerdo con las bases y la ordenación de la actuación económica general... la competencia exclusiva sobre las siguientes materias: 1a) fomento y planificación económica en Andalucía ...". En esta disposición se ha basado el gobierno andaluz para elaborar los distintos planes de desarrollo. 


\section{CUADRO 1}

\section{Objetivos de los diferentes planes}

\begin{tabular}{|c|c|c|}
\hline PLAN & $\begin{array}{l}\text { OBJ ETIVOS } \\
\text { FINALES }\end{array}$ & $\begin{array}{c}\text { OBJ ETIVOS INTERMEDIOS } \\
\text { O ESTRATEGIAS }\end{array}$ \\
\hline $\begin{array}{l}\text { PLAN } \\
\text { ECONÓMICO } \\
\text { PARA } \\
\text { ANDALUCÍA } \\
(\mathbf{1 9 8 4 - 1 9 8 6 )}\end{array}$ & $\begin{array}{l}\text { - Reducción del paro } \\
\text { - Desarrollo equilibrado } \\
\text { - Distribución másjusta }\end{array}$ & $\begin{array}{l}\text { - Aumento mayor economía nacional } \\
\text { - Integración sectorial } \\
\text { - Integración espacial } \\
\text { - Equipamientos colectivos }\end{array}$ \\
\hline $\begin{array}{l}\text { PROGRAMA } \\
\text { ANDALUZ DE } \\
\text { DESARROLLO } \\
\text { ECONÓMICO } \\
(1987-1990)\end{array}$ & $\begin{array}{l}\text { - Creación de empleo } \\
\text { - Aumento de la renta e } \\
\text { integración del siste- } \\
\text { ma productivo } \\
\text { - Mejora calidad devida }\end{array}$ & $\begin{array}{l}\text { - Prioridades sectoriales } \\
\text { - Innovación y desarrollo tecnológico } \\
\text { - Formación capital humano } \\
\text { - Ampliación mercados } \\
\text { - Proyección territorial }\end{array}$ \\
\hline $\begin{array}{c}\text { PLAN } \\
\text { ANDALUZ DE } \\
\text { DESARROLLO } \\
\text { ECONÓMICO } \\
(1991-1994)\end{array}$ & $\begin{array}{l}\text { - Crecimiento sosteni- } \\
\text { ble y equilibrado } \\
\text { - Mejora calidad devida } \\
\text { y redistribución }\end{array}$ & $\begin{array}{l}\text { - Articulación producción y territorio } \\
\text { - Revalorización sectores y fomento } \\
\text { actividad } \\
\text { - Medio ambiente y equilibrio } \\
\text { territorial } \\
\text { - Objetivos sociales y culturales }\end{array}$ \\
\hline $\begin{array}{l}\text { PLAN } \\
\text { ECONÓMICO } \\
\text { ANDALUCÍA } \\
\text { HORIZONTE } \\
2000 \\
(1998-2000)\end{array}$ & $\begin{array}{l}\text { - Desarrollo económico } \\
\text { y social favorable a la } \\
\text { generación de empleo }\end{array}$ & $\begin{array}{l}\text { - Desarrollo solidario } \\
\text { - Desarrollo equilibrado } \\
\text { - Desarrollo sostenible } \\
\text { - Competitividad } \\
\text { - Desarrollo comercial } \\
\text { - Ampliación y diversificación de la } \\
\text { base productiva }\end{array}$ \\
\hline $\begin{array}{l}\text { PLAN } \\
\text { ECONÓMICO } \\
\text { ANDALUCÍA } \\
\text { SIGLO XXI } \\
(\mathbf{2 0 0 2 - 2 0 0 5 )}\end{array}$ & $\begin{array}{l}\text { - Avanzar en una socie- } \\
\text { dad próspera y sin ex- } \\
\text { clusiones, mantenien- } \\
\text { dolatrayectoriadecon- } \\
\text { vergenciareal connues- } \\
\text { tro entorno europeo, } \\
\text { con el empleo como } \\
\text { principal referente }\end{array}$ & $\begin{array}{l}\text { - Alcanzar una competitividad } \\
\text { sostenible } \\
\text { - Conseguir el crecimiento diferen- } \\
\text { cial sostenido } \\
\text { - Convergencia }\end{array}$ \\
\hline
\end{tabular}

Fuente: Planes de desarrollo de Andalucía. 
incidir sobre la realidad económica. Ello no impide que se vayan estableciendo cambios graduales para adaptarse a las variaciones de la realidad, pero nunca cambios bruscos de orientación. Las variaciones observadas de objetivos intermedios pueden responder en parte a estas adaptaciones razonables, pero en general parece que existe una falta de estabilidad estratégica entre los distintos planes. ${ }^{7}$

\section{La elaboración y la ejecución del plan}

Los aspectos administrativos del Plan parten de la experiencia acumulada en los otros planes, y en general se pueden valorar positivamente. ${ }^{8}$

La elaboración del Plan pivotó alrededor de la Dirección General de Planificación de la Consejería de Economía y Hacienda; fue la encargada de la realización de los trabajos y estudios y de la coordinación de los trabajos de las distintas Consejerías.

Ha existido una Comisión Técnica de Redacción presidida por el Secretario general de Economía de la Consejería de Economía y Hacienda, en la que participaron también representantes de las otras Consejerías.

Asimismo ha existido también un Comité de expertos para articular la participación del mundo científico y universitario.

Finalmente la colaboración de las corporaciones locales y de los agentes económicos y sociales se canalizó a través de la Comisión de Planificación Económica de Andalucía.

El documento del Plan fue aprobado por el Parlamento de Andalucía.

En cuanto a la ejecución del Plan, la normativa es bastante escueta, se limita a trasformar la Comisión Técnica de Redacción en una Comisión de Seguimiento. En el propio documento se indica que los órganos ejecutores presentarán a la Comisión informes anuales. La evaluación periódica corresponde al Consejo de Gobierno.

7 Un análisis comparativo de los planes exigiría la consideración de las líneas de actuación, pero no es posible dada la dimensión de este escrito. El número de líneas en el Plan Siglo XXI es de 132; ello da idea del contenido de este documento.

8 Se puede ver el Decreto 409/2000, de 24 de octubre, por el que se acuerda la formulación del Plan Económico de Andalucía Siglo XXI y se regula su proceso de elaboración. 
Unos breves comentarios:

a) Respecto a la elaboración, es discutible si la concreción de las distintas acciones responde a una instrumentación de los objetivos del Plan, o si es una mera incorporación de políticas autónomas de las distintas Consejerías.

b) Realmente la primera opción del párrafo anterior representaría que el Plan determine la mayor parte de la política de la J unta de Andalucía, lo cual no creemos que responda a la realidad. En este caso, la elaboración y la ejecución del Plan debería asignarse a la Presidencia de la lunta y no a una consejería concreta. Pensamos que la evaluación por el Consejo de Gobierno va en el camino adecuado, pero nos parece insuficiente.

c) En cuanto a la evaluación final, se basa en un informe elaborado al finalizar el período de vigencia del Plan, que contendrá una valoración de sus efectos a medio plazo; el desarrollo de este informe se realizará por la Dirección General de Planificación. Dentro de sus respectivas competencias, la evaluación final será objeto de deliberación en el Consejo de Gobierno y en el Parlamento de Andalucía, aunque esto no aparezca muy claramente en el documento.

Consideramos de la máxima trascendencia esta fase de la planificación y nos tememos que no se le déla necesaria relevancia. El control dela ejecución hará que el Plan sea o no sea una guía decisiva de la política del gobierno andaluz.

También es importante el conocimiento del Plan en los medios ciudadanos. La colaboración de las empresas, de los organismos y de los andaluces en general pueden hacer del Plan un documento vivo. Cuando se aprobó el Plan Económico para Andalucía 1984-1986, se hizo una presentación pública del mismo en las provincias andaluzas, por lo que se habló del Plan y se involucró a los medios andaluces en su desarrollo. En nuestra opinión, en esta ocasión, ha faltado ese dar a conocer la planificación y puede ser que, como en casos anteriores, la evaluación final pase sin pena ni gloria por la CA andaluza.

Una anécdota en este sentido: cuando se aprobó el Plan Siglo XXI, el Observatorio Económico de Andalucía, una asociación independiente, criticó la estrategia contenida en este programa, afirmando incluso que la J unta carecía de estrategia. Ello motivó una agria disputa entre la Consejería de 
Economía y el Presidente del Observatorio. No creemos que ésta sea la forma más adecuada de dar a conocer el Plan, por parte de la Administración andaluza.

\section{Su relación con otros planes}

La Junta de Andalucía y otros organismos públicos tienen otros planes, programas o documentos que pueden afectar a la planificación y con los que es necesario establecer una cierta coherencia.

Destacamos en primer lugar, el V Acuerdo de Concertación Social, suscrito con los agentes económicos y sociales. El contenido del Acuerdo fue tenido en cuenta en la elaboración del Plan ${ }^{9}$, lo que responde a esa necesaria coherencia que hemos citado.

Menos coherente parece el que los Foros de la segunda modernización, impulsados por el propio Presidente de la J unta, se aprueben en el año 2003, sin tener en cuenta el Plan. Ciertamente en algunos de sus apartados se incluyen aspectos de esa llamada sociedad del conocimiento, pero creemos que la reciente propuesta del Presidente representa un cambio de orientación que varía, en parte, el contenido del Plan.

Existen también otros documentos: planes sectoriales, etc., cuya lógica no siempre coincide con la del proceso planificador.

Otros niveles, el estatal y el europeo, deberían estar presentes en el documento. La política del gobierno central y de la Unión Europea son factores a tener en cuenta. Ambos aspectos aparecen en el escenario financiero, lo cual es adecuado pero insuficiente; la política de estabilidad presupuestaria y los programas operativos tienen algo que ver con el contenido del Plan, no siendo meras limitaciones financieras.

\section{Algunos aspectos positivos del plan}

Destacaríamos en primer lugar el diagnóstico de la economía andaluza. En varios extensos capítulos se realiza un excelente análisis, que constituirá, sin

9 Decreto 264/2001 de 4 de diciembre, por el que se modifica el Decreto 409/2000, de 24 de octubre, por el que se acuerda la formulación del Plan Económico Andalucía Siglo XXI y se regula su proceso de elaboración. 
duda, una referencia que habrá que citar cuando se quiera estudiar la econo mía andaluza. Para establecer la estrategia de desarrollo se ha utilizado un análisis DAFO, que es una buena ayuda para definir la estrategia en términos de competitividad sostenible.

También habría que subrayar que, tanto en el diagnóstico como en las políticas instrumentales, se contemplan todos los aspectos económicos y sociales que forman parte de la CA. Un par de ejemplos: los problemas del cambio climático y los de las migraciones no están ausentes del Plan.

La minuciosidad con que se recogen las políticas instrumentales y las líneas de actuación dan un contenido muy completo al Plan y un nivel adecuado de coherencia interna; valoración positiva, aunque como hemos indicado en el apartado anterior, nos preguntamos si la iniciativa parte del organismo planificador o de las distintas consejerías.

\section{Algunos aspectos negativos}

La falta de continuidad, ha sido a veces un defecto de la planificación de la unta de Andalucía. Así, entre el tercer y el cuarto plan, hay un lapso de tres años que no están cubiertos. Entre el cuarto plan y el actual hay un hueco de un año, que fue motivado por la aprobación del V Acuerdo de Concertación Social. Además del Plan Siglo XXI debería haber comenzado el año 2002, pero no fue aprobado hasta noviembre de dicho año, lo que deja un año más sin programa.

La ausencia de una evaluación de los planes anteriores en el propio documento acentúa esta falta de continuidad. Parece como si una vez aprobado el plan, éste dejara de estar presente en la política andaluza.

Por otra parte, si se quiere evitar un nuevo centralismo, ahora de carácter regional, hace falta una especie de pacto local, que dé un papel a las corporaciones locales en el proceso planificador, y en otras políticas, claro está. La política de cooperación con las corporaciones locales, incluida en el Plan, es buena pero no responde al planteamiento que proponemos. ${ }^{10}$

10 Las corporaciones locales están representadas en la Comisión de Planificación de Andalucía; sin embargo, la distribución de competencias respecto a la planificación, no permiten afirmar que de esta forma se haya asegurado la intervención de las corporaciones locales en la elaboración del Plan. 
Un aspecto formal: en otros planes aparecía en cada política o línea el organismo ejecutor, pero no así en este Plan. Ello nos parece indispensable y no sólo por razones formales.

\section{La financiación y programación}

El documento incluye unos cuadros que recogen la financiación del Plan. Los supuestos de partida son bastante razonables aunque ignoramos el cuadro macroeconómico de la economía andaluza en el que debe basarse; pensamos que hubiera sido más realista haber considerado distintos supuestos o escenarios, o incluso haber realizado un análisis de sensibilidad.

En la distribución por políticas, se da prioridad al gasto corriente y de inversión en la política de cualificación de recursos humanos, en primer lugar, y después a la de transportes, complejo agroalimentario, servicios sociales y medio ambiente. Esta priorización indica cuál es la filosofía del Plan, que nos parece adecuada en líneas generales; quizás podría considerarse escasa la asignación a la industria, no agroalimentaria, lo que contrasta con algunas otras propuestas de planificación del gobierno andaluz.

Lógicamente la distribución por políticas habrá contado con la asignación de recursos a las distintas líneas de actuación, sin embargo ello no se recoge en el texto. Este dato es importante a la hora de ejecutar el Plan, aunque sea con una razonable flexibilidad.

\section{Aspectos formales}

El documento del Plan está muy bien editado. Con un lenguaje claro y preciso, no abusando de tecnicismos, cumple su función de dar a conocer este importante proceso.

La edición es bastante austera, evitando lujos impropios de un organismo público.

El libro mantiene una gran coherencia entre los distintos capítulos que citamos al principio. Sólo echamos en falta una breve relación bibliográfica, que incluyera al menos la referencia a los anteriores planes de la CA. 


\section{Opinión de conjunto}

Hemos valorado positivamente algunos aspectos del Plan y criticado algunos otros. En nuestra opinión es importante que se planifique en nuestra CA. Un plan determina en todo caso una cierta coherencia de las políticas de los distintos departamentos; asimismo puede influir en otros planes o programas, aunque no siempre sea así.

El trabajo realizado por la Dirección General de Planificación, y en menor medida, por otros organismos, nos parece valioso.

En resumen, es bueno que se haya elaborado este Plan, que en alguna medida contribuirá positivamente a mejorar la economía andaluza. Lo que nos parece discutible es el lugar que ocupa la planificación en la política de la Junta, pero ése es otro problema.

\section{Bibliografía}

Junta de Andalucía, (1985), Plan Económico Para Andalucía (1984-1986), Sevilla, Ed. Consejería de Economía, Planificación, Industria y Energía.

- (1988), Programa Andaluz de Desarrollo Económico (1987-1990), Sevilla, Ed. Consejería de Economía y Fomento.

- (1991), Plan Andaluz de Desarrollo Económico (1991-1994), Sevilla, Ed. Consejería de Economía y Hacienda.

- (1998), Plan Económico Andalucía Horizonte 2000 ( 1998-2000), Sevilla, Ed. Consejería de Economía y Hacienda.

- (2003), Plan Económico Andalucía Siglo XXI (2002-2005), Sevilla, Ed. Consejería de Economía y Hacienda. 